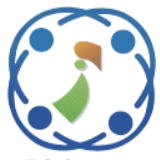

\title{
Palm Vein Identification Based on Hybrid Feature Selection Model
}

\author{
Ali Saeed Alfoudi ${ }^{1,2 *}$ \\ Ali Hakem Alsaeedi ${ }^{1}$ \\ Mohammed Hamzah Abed ${ }^{1}$ \\ Abayomi M. Otebolaku \\ Yasmeen Sajid Razooqi ${ }^{1}$ \\ ${ }^{1}$ University of Al-Qadisiyah, College of Computer Science and Information Technology, Iraq \\ ${ }^{2}$ Liverpool John Moores University, College of Computer Science, United Kingdom \\ ${ }^{3}$ Department of Computing, Faculty of Science, Technology and Arts, Sheffield, United Kingdom \\ * Corresponding author's Email: a.s.alfoudi@qu.edu.iq
}

\begin{abstract}
Palm Vein Identification (PVI) is a modern biometric security technique used for enhancing security and authentication systems. The key characteristics of palm vein patterns include its uniqueness to each individual, its unforgettability, non-intrusiveness and its ability for disallowing unauthorized persons. However, the extracted features from the palm vein patterns are huge with high redundancy. In this paper, we propose a combined model of two-Dimensional Discrete Wavelet Transform, Principal Component Analysis (PCA), and Particle Swarm Optimization (PSO) (2D-DWTPP) that feeds wrapper model with an optimal subset of features to enhance the prediction accuracy of -palm vein patterns. The 2D-DWT extract features from palm vein images, using the PCA to reduce the redundancy in palm vein features. The system has been trained to select high recognition features based on the wrapper model. The proposed system uses four classifiers as an objective function to determine PVI which include Support Vector Machine (SVM), K Nearest Neighbor (KNN), Decision Tree (DT) and Naïve Bayes (NB). The empirical results proved that the proposed model has the best results with SVM. Moreover, our proposed 2D-DWTPP model has been evaluated and the results show remarkable efficiency in comparison with AlexNet and other classifiers without feature selection. Experimentally, the proposed model has better accuracy as reflected by $98.65 \%$ whereas AlexNet has $63.5 \%$ accuracy and the classifier without feature selection process has $78.79 \%$ accuracy.
\end{abstract}

Keywords: Palm vein identification, 2D-Dwt, Feature selection, Particle swarm optimization, Feature selection.

\section{Introduction}

The unprecedented growth of biometric-based identity management systems pushes governments and private stakeholders to look for more efficient management mechanisms, that has unique physiological or behavioral traits to improve the people's identification and verification [1]. The main problem in this work is security issues and using biometric method to achieved high security than other models. The establishments such as airports or bank transmit very important and serious data, therefore it requires a strict security system. The traditional security methods based on PIN, magnetic cards, keys and smart cards have become insufficient and week [2]. Moreover, the biometric system appears as a promising solution to improve their security system. A biometric system is a recognition system that allows identifying a certain physiological or behavioral characteristic of individual traits from images (dataset) in order to extract different features [3]. Recognition of persons using vital factors, includes biometric such as fingerprint, face, iris, palm print, retina, palm vein, hand geometry, voice, signature and gait, has become an urgent necessity to improve biometric security systems. The limitations of using some vital characteristics are significantly suffering from default of extracted features due to disease or damage in their functions [4]. The people who suffer from the disease or have manual work, their fingerprint features might be impossible to extract. In addition, the face-based recognition model still suffers from limitations in terms of stability and lighting. All biometrics could be valid for a dead person or severed human limbs except the 
palm veins [5]. Palm vein identification method, which provides a high possibility of recognition. Palm vein is a better choice of biometrics because its features are deep and non-superficial. Therefore, palm vein is difficult to manipulate or change $[4,6]$. Moreover, the palm vein patterns are also a difficult method to forge because it is internal in the body and it cannot be valid with the dead parts. Taking into account the aforementioned requirements of palm vein, we propose a hybrid model that consists of $2 \mathrm{D}$ wavelet coefficient and particle swarm optimization methods. The model mechanism aims to ensure the optimization of feature selection of palm vein. The results show, the proposed model enhances the prediction of query of machine learning algorithms, where in the proposed system with SVM the classification accuracy data reaches to 98.65 for lefthand and 98.40 for right-hand. Additionally, the proposed model works affective with high dimensional features to select unique attribute.

The rest of this paper is organized as follows. Section 2 presents the related work with palm vein preprocessing, verification and identification. The proposed model and its components discuss in Section 3. The experimental results and analysis explain in Section 4. Finally, the conclusion and future works present in Section 5.

\section{Related work}

Recently, in the field of palm vein research, many methods have been proposed for recognition and classification to create a biometric system based on palm vein pattern. All the researchers model mainly consists of three parts: preprocessing, features extraction and classification or recognition phase.

In other work [2], the researcher suggested palm vein recognition model based on Gabor filter as a feature extraction and proposed set of steps for preprocessing phase starting with adaptive histogram equalization for adjusting the intensity of images, then using Discrete Fourier transform, the coefficient multiply by Gaussian high pass filter. Also, the author used Euclidian distance as matching phase. Moreover, the proposed model achieved good results but still suffering from some limitations in term of complex features and wide range of dataset unlike machine learning techniques have a flexible handing with complex features extraction. In [4], the authors suggested palm vein verification based on Multiple features extracted based on local and global features using wavelet and local binary pattern. Moreover, they used Gaussian matched filter with enhancement part. In the last stage, they utilized Manhattan distance as a matching method, where, the minimum distance method used to find the similarity between training and testing data. The researchers in [7] proposed a new method for palm recognition by combing two different methods DAISY descriptor and the Coarse-to-fine Patch Match (CPM) algorithm. In [8] authors detected the region of interest based on fast ROI algorithm, then normalize the brightness using adaptive histogram equalization, to prepare palm vein images to be ready for features extraction and classification using Convolutional Neural Network. In reference [9], R. Meitram and P. Choudhary proposed Otsu thresholding to estimate palm region by binaries the images to segment region of interest, then using 2D Gabor filter for features extraction as well as using Artificial Neural Network for classification and matching phase. In [10], the authors used palm vein for biomedical identification, the palm images are processing by palm boundary detection and Competitive Hand Valley Detection (CHVD) algorithm is used to get reference points for ROI in the cropped image, then used local derivative pattern for features extraction from each pixel of image, in the matching phase two methods are tested histogram intersection and decision making. In [11] the researchers proposed recognition method based on learning vector Quantization, the images acquisition then preprocessing the images to improve the smoothing of images and detection the region of interest. Next, from ROI the features extracted using peak point detection of palm, finally the training done by using Learning Vector Quantization. In [12], Y. Zhou and A. Kumar proposed a model for human identification using palm vein images. The images preprocessed using contrast stretching and histogram equalization, the enhanced palm vein images were used to extract the features the researcher suggested two methods: Neighborhood Matching Radon Transform and Hessian-Phase-Based Feature Extraction.

In [13], the authors proposed a multimodal biometric system using vascular patterns of the hand such as finger vein and palm vein images. Initially, the input palm vein and finger vein images preprocessed make them suitable for further processing. Subsequently, the features from palm and finger vein images are extracted using a modified twodimensional Gabor filter and a gradient-based technique. These extracted features matched using the Euclidean distance metric, and they fused at the score level using the fuzzy logic technique, both matching methods worked with simple features to give high accuracy and to be fast. In [14] ,The researcher proposed model based on 2D wavelet transform for features extraction and minimum distance to find the similarity between training and 
testing vein images. In reference [15] the authors proposed and analyzed two novel CNN, they investigated the influence of some image quality enhancement methods, such as contrast adjustment and normalization, Gaussian smoothing, contrast limited adaptive histogram equalization, and Hessian matrix based coarse vein segmentations.

Most of the above mentioned studies are focusing on raw of local and global features of dataset, where the methods of these studies are suffering from selected all features in the dataset, which is some of them are not unique.

Unlike the aforementioned studies, our proposed solution considers features reduction and selection, which it uses PSO and PCA to select only corresponding features. Therefore, the proposed solution achieves better accuracy result.

Finally, Table 1 shows the summarized each step of the verification model for the related works studies:

Table 1. Summary of related work

\begin{tabular}{|c|c|c|c|}
\hline \multirow{2}{*}{ Author } & \multicolumn{3}{|c|}{ Method description } \\
\hline & Pre-processing & Feature extraction & Matching/classification \\
\hline [2] & $\begin{array}{l}\text { adaptive histogram equalization, } \\
\text { Discrete Fourier transform }\end{array}$ & Gabor filter & Euclidian distance \\
\hline [4] & Gaussian matched filter & $\begin{array}{l}\text { two-dimensional wavelet } \\
\text { transform and LBP }\end{array}$ & Manhattan distance \\
\hline [7] & $\begin{array}{l}\text { ROI segmentation } \\
\text { Intensity normlization }\end{array}$ & DAISY descriptor & CPM matching \\
\hline [8] & $\begin{array}{l}\text { Fast ROI , adaptive histogram } \\
\text { equalization }\end{array}$ & $\mathrm{CNN}$ & $\begin{array}{l}\text { Train Convolutional Neural } \\
\text { network }\end{array}$ \\
\hline [9] & Otsu thresholding & 2D Gabor filter & Artificial Neural Network \\
\hline$[10]$ & boundary detection, CHVD & local derivative pattern & $\begin{array}{l}\text { histogram intersection and } \\
\text { decision making }\end{array}$ \\
\hline$[11]$ & Palm boundary and extract RIO & peak point detection & $\begin{array}{l}\text { Learning Vector } \\
\text { Quantization }\end{array}$ \\
\hline [12] & $\begin{array}{l}\text { contrast stretching and histogram } \\
\text { equalization }\end{array}$ & $\begin{array}{l}\text { Neighborhood Matching } \\
\text { Radon Transform and } \\
\text { Hessian-Phase-Based } \\
\text { Feature Extraction }\end{array}$ & $\begin{array}{l}\text { Matching based on minimum } \\
\text { coefficient }\end{array}$ \\
\hline [13] & Enhancement filters & $\begin{array}{l}\text { two-dimensional Gabor } \\
\text { filter and a gradient-based } \\
\text { technique }\end{array}$ & $\begin{array}{l}\text { Euclidean distance and fuzzy } \\
\text { logic }\end{array}$ \\
\hline$[14]$ & Low pass Gaussian filter & $\begin{array}{l}\text { two-dimensional wavelet } \\
\text { ransform }\end{array}$ & cosine minimum distance \\
\hline$[15]$ & $\begin{array}{l}\text { contrast adjustment Gaussian } \\
\text { smoothing, contrast limited adaptive } \\
\text { histogram equalization, and Hessian } \\
\text { matrix }\end{array}$ & $\mathrm{CNN}$ & $\mathrm{CNN}$ \\
\hline $\begin{array}{l}\text { The } \\
\text { Proposed } \\
\text { model }\end{array}$ & $\begin{array}{l}\text { adaptive histogram equalization to } \\
\text { enhace the apperance then used } \\
\text { negative images }\end{array}$ & $\begin{array}{l}\text { Hybrid using } \quad \text { D } \\
\text { DWT ,with features } \\
\text { selection PSO and PCA }\end{array}$ & SVM \\
\hline
\end{tabular}

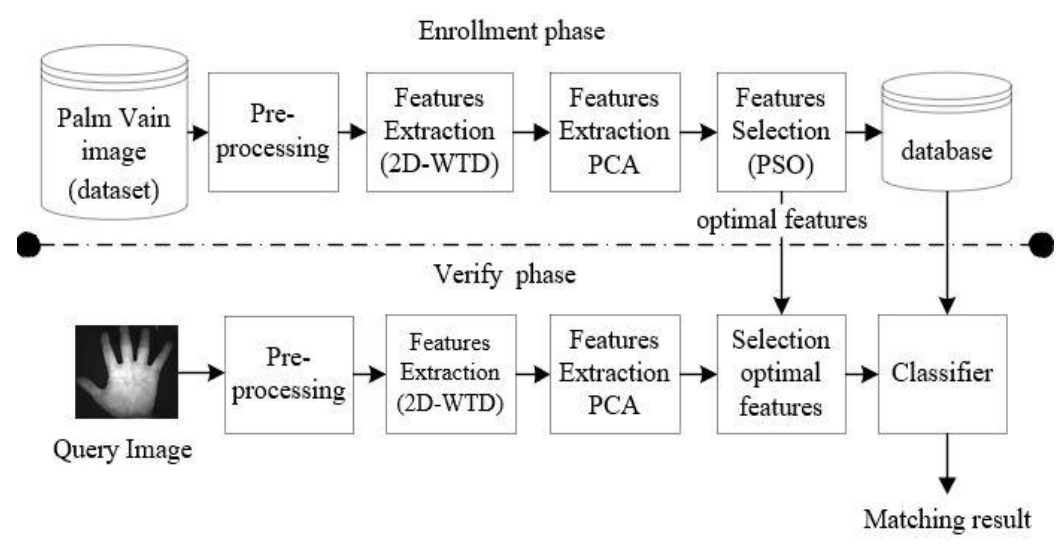

Figure. 1 Proposed model structure 


\section{The proposed 2D-DWTPP model}

The proposed model for palm vein identification model mainly consists of three phases: palm vein images preprocessing, feature extraction, reduction, and classification. Fig. 1 illustrates the details of the proposed model.

\subsection{Data preprocessing}

The first phase of our model is the preprocessing of palm vein images. It's used to enhance the images to make the palm's vessel more visible. The proposed model investigates two steps. The first step is the conversion of the vein image into grayscale enhancement using adaptive histogram equalization to improve the quality of images and to make vessels visible. The second step of the preprocessing phase is the conversion of images into a negative image to show the vessels clearly and it this is used as a final output of palm image of the preprocessing phase. Fig. 2 illustrates the steps of the preprocessing stage of the proposed model.

\subsection{Feature extraction ( $2 \mathrm{D}$ wavelet)}

The feature extraction phase aims to extract the relevant features of the palm vein patterns before moving to the recognition phase. The features are extracted from 2 levels of HARR two-dimensional wavelet coefficient as a raw feature. In order to be able to perform palm vein recognition (PVR), one of the key processes is the extraction of different vein patterns. The wavelet function is used as an efficient
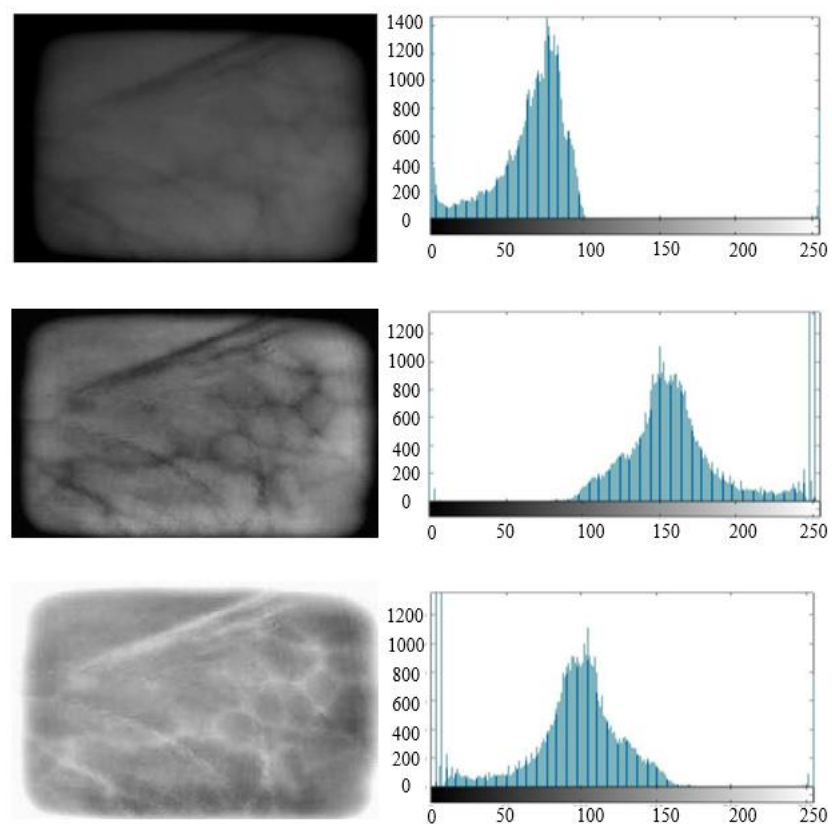

Figure. 2 Preprocessing steps with histogram of each stage

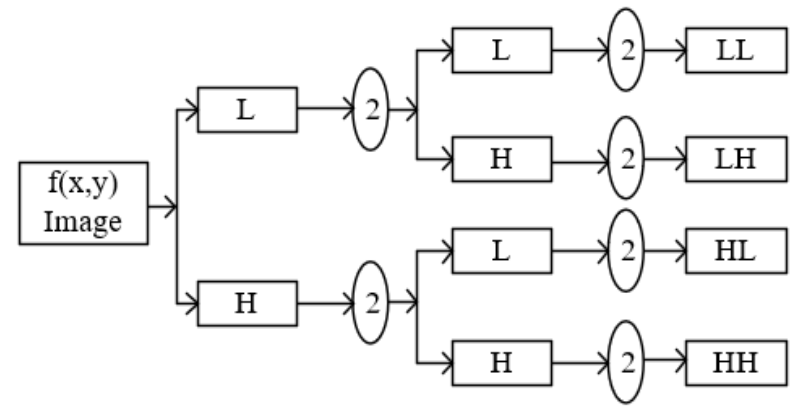

Figure. 3 Decomposition of 2D DWT [2]
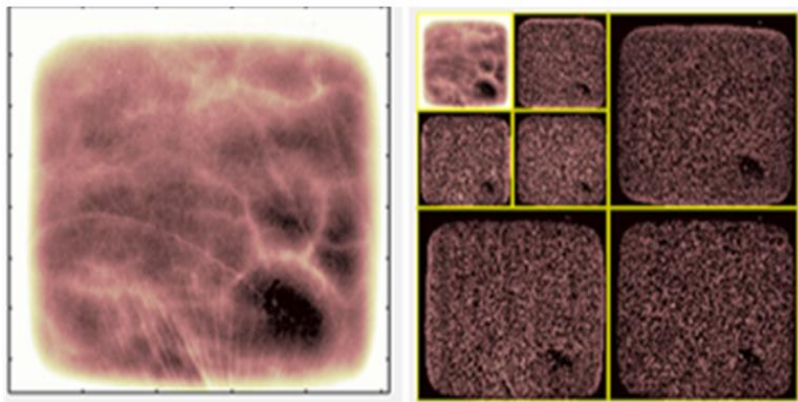

Figure. 4 Palm vein and 2 level haar wavelet

method for representing the image, it's surface and curve [13]. The discrete wavelet transforms (DWT) is a very common technique for analysis of nonstationary signals $[16,17]$. The basic idea of using DWT is for the representation of a signal as a series of low-pass and high pass approximations. The low pass version corresponds to the signal, whereas the highpass version corresponds to the details. This is done at different resolutions. It is almost equivalent to filtering the signal with a bank of bandpass filters whose impulse responses are all roughly given by scaled versions of a mother wavelet [18].

Fig. 3 and 4 show the two-level composition of an image into four components called 4 sub-images of the wavelet transform to low level coefficients of wavelet considered as a raw feature. It uses the Principal Component Analysis (PCA) to reduce the number of features and the redundancy to find a linear set of features that classify into two or more classes of object or event [19]. The main reason for using 2D Haar DWT for features extraction is that it gives us high information and features of images and all the details of vein vessels.

\subsection{Feature reduction based on PCA}

The extracted features from the palm vein pattern are huge with high redundancy. Furthermore, the applied wrapper model on redundancy feature is inefficient because it is based on the selection of features randomly [19]. Therefore, reducing features redundancy is the necessary to enhance the wrapper model. In this paper, the Principle Component 
Analysis (PCA) is used for feature reduction. It is an orthogonal linear transformation technique that transfers raw data into new form hopefully equal or fewer dimensions of original data [20]. Furthermore, it is used as features reduction and extraction method in data science. Technically, PCA calculates the eigenvectors of a covariance matrix where the highest eigenvalues represent the significant features.

\subsection{Particle swarm optimization (PSO)}

PSO is an Evolutionary Algorithms (EA) that was inspired by animal social behaviors that live in groups [21]. PSO is preferred over other EA because it has a simple mathematical model with the minimum number of variables. Structurally, it works like the evolutionary optimizers by starting randomly and using all particles to find the optimal solution [22]. The search processing of PSO is technically based on groups of particles. Each particle has its velocity and position, the velocity and position are updated dynamically during search progress. The velocity has been added to the position to generate a new solution. The best solution of PSO's particle is called the local best optimum $\left(p_{\text {best }}\right)$. The re best solution $\left(g_{\text {best }}\right)$ is the best choice among $p_{\text {best }}$ 's and is updated after reaching the optimizing iteration. Eq. (1) computes the velocity of particles [23].

$$
\begin{gathered}
V_{i}^{d}(t+1)=w(t) V_{i}^{d}(t)+c_{1} r_{1}\left(\text { pbest }_{i}^{d}-\right. \\
\left.x_{i}^{d}(t)\right)+c_{2} r_{2}\left(\text { gbest }^{d}-x_{i}^{d}(t)\right)
\end{gathered}
$$

where: $r 1$ and $r 2$ are random variables in the range [0, 1], $c 1$ and $c 2$ are positive constants, $w$ is the inertia weight, $v_{i}^{d}(t), x_{i}^{d}(t)$ indicate the velocity and position of $i^{t h}$ particle at iteration $t$ in $d^{t h}$ dimension, respectively.

The PSO uses Eq. (2) to find the new value of particle position (candidate solution) [23].

$$
x_{i}^{t+1}=x_{i}^{t}+v_{i}^{t+1}
$$

where: $x_{i}^{t}$ is old particle value, and $x_{i}^{t+1}$ is a new particle value.

\subsection{Wrapper model}

Wrapper model is a feature selection method that tests deferent groups of features and then selects the group that with the best result [24]. It has selected the features based on randomness. The metaheuristic techniques which are very successful in applying randomness for search on an optimal solution. Therefore, it significantly optimizes the selection of

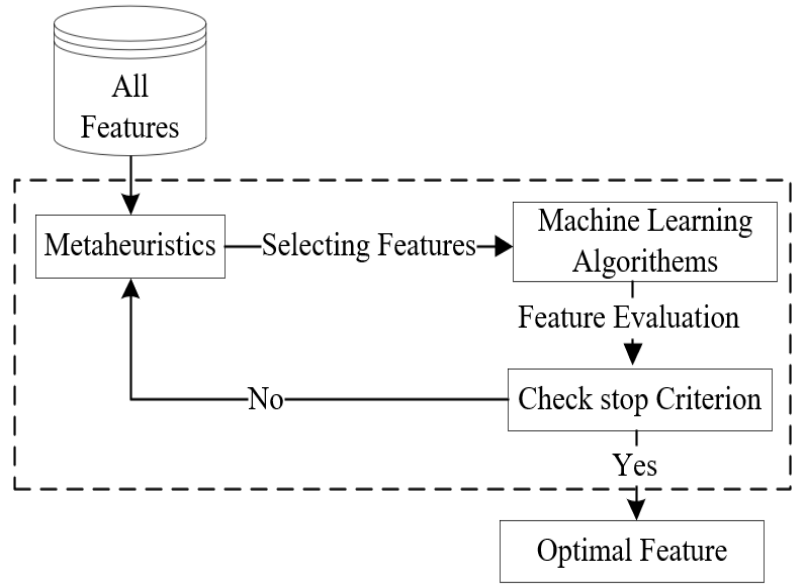

Figure. 5 Features selection based on Wrapper model and Metaheuristic

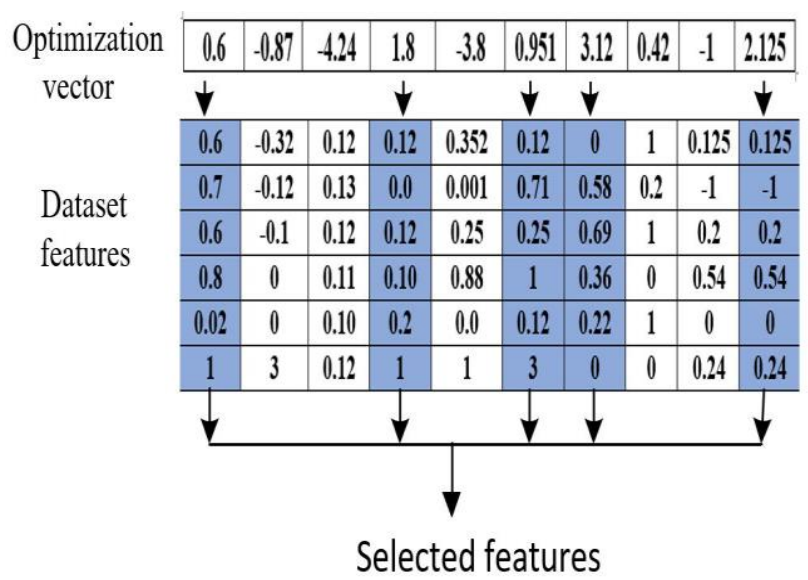

Figure. 6 Features selecting with threshold 0.5

features that feed into the wrapper model. Fig. 5 illustrates the principles of the wrapper model with metaheuristic for selecting the optimum feature.

Generally, the wrapper model is based on binary feature selection- only features that correspond to 1 in optimization algorithm vector are selected. It restricts the metaheuristic algorithms to search in limited boundaries of either 0 or 1 [23]. The extending search space is enhanced performance of metaheuristic algorithms. The applied space searching interval range is $[-5,+5]$ in the proposed system [25]. The threshold for feature selection is determined by the user for each experiment. Fig. 6 illustrates the encoding and selecting features.

The wrapper model selects the reverent features based on metaheuristic optimization technique. Technically, it is a greedy optimization algorithm which aims to find the best feature subset group by calculating the accuracy of each group [20]. It repeatedly searches on optimal subset features until it reaches to determinants - stop criteria - of optimization operation [22]. 


\subsection{Classification}

In this research four object classifiers have been used as an object function to determine the pattern of palm vein.

\section{- K Nearest Neighbors (KNN)}

$\mathrm{K}$ nearest neighbors $(\mathrm{KNN})$ is a non-parametric technique used in supervised machine learning to classify new object based on the high-density class of the nearest available cases. Generally, the distance between objects is calculated by one of Minkowski Distances (Manhattan, Euclidean Distance, and Distance), in this work the Euclidean distance Eq. (3) has been applied to find the distance.

$$
d_{x, y}=\sqrt{\sum_{i=1}^{n}\left(x_{i}-y_{i}\right)^{2}}
$$

\section{- Support Vector Machine (SVM)}

A Support Vector Machine (SVM) is a supervised machine learning introduced by [24] as both regression and classifier model. Technically, it classifies a new object based on Hyperplane and Support Vectors. The hyperplane is multiple lines detected the boundaries of classes that help to determine the class the data objects easily. SVM model sets the diminution of hyperplane based on features present in the dataset [23]. Eq. (4) calculates the hyperplane.

$$
w \cdot x+b=0
$$

where: $x$ is input, $w$ is weights vector, and $b$ is bias. The dataset that has few numbers of features often is linearly separable, therefore, The SVM uses Eq. (5) to classify a new object $x$.

$$
f(x)=\operatorname{sign}(w \cdot x+b)
$$

The high dimensional data potentially is not in every case linearly separable as a result, the nonlinear decision function Eq. (6) is used to classify a new object $x$.

$$
f(x)=\operatorname{sign}\left(\sum_{i=1}^{N} a_{i} y_{i} K\left(x_{i} \cdot x\right)+b\right)
$$

where: $K\left(x_{i} \cdot x\right)$ is the Kernel function, $y_{i}$ is class data.

- Decision Tree (DT)

A Decision Tree (DT) is a non-parametric supervised machine learning model that predicts the class of query data based on a sequences series of decision rules [26]. The root of each decision rule is a feature of data has the highest information gained than others. Eq. (7) calculates information gain of feature $A$ for dataset $D$.

$$
\begin{gathered}
\operatorname{Gain}(A)=\sum_{i=1}^{n} A_{p_{i}} \log _{2}\left(A_{p_{i}}\right)- \\
\sum_{i=1}^{m^{2}} d_{p_{i}} \log _{2}\left(d_{p_{i}}\right)
\end{gathered}
$$

where $d_{p_{i}}$ is the probability of each class in the dataset, $A_{p_{i}}$ probability of each distinct entity in feature $A$.

- Naïve Bayes (NB)

Naive Bayes is a probabilistic supervised machine learning model predicts the category of query data based on core concepts Bayes' theorem [25]. Technically, it calculates the probability $P(y / x)$ of query data $x$ with all training classes $y$. The high $P(y / x)$ is determined by the new class of $x$. Eq. (8) calculates the class probability, for more details refers to reference [27].

$$
P\left(y \mid x_{1}, \ldots, x_{n}\right)=\frac{P\left(x_{1} \mid y\right) P\left(x_{2} \mid y\right) \ldots P\left(x_{n} \mid y\right) P(y)}{P\left(x_{1}\right) P\left(x_{2}\right) \ldots P\left(x_{n}\right)}
$$

$P\left(x_{1} \mid y\right)$ is calculated by Gaussian Naive Bayes Eq. (9).

$$
P\left(x_{i} \mid y\right)=\frac{1}{\sqrt{2 \pi \sigma_{y}^{2}}} \exp \left(-\frac{\left(x_{i}-\mu_{y}\right)^{2}}{2 \sigma_{y}^{2}}\right)
$$

where $\mu_{y}, \sigma_{y}$ is mean and standard deviation of train class $y$.

\section{Experiments and result analysis}

We have evaluated the performance of palm vein image classification based on standard dataset PUT Vein Database. Then using 2D Haar Wavelet 2 level as the feature extraction from the entire image. The experimental test result based on raw features and reduction of features using PCA is compared, and the results are presented in the next sections.

\subsection{Put vein dataset}

The palm vein image dataset that we used in our experiments [5, 28]. It contains 1200 human vein pattern images for left and right hands; the researcher collected the data from 50 persons for both hands. The images have been taken in three sessions, four images each time, for at least one week between each session. The image in the database has $1280 \times 960$ resolution and saved as a 24-bit bitmap. 


\subsection{Result and experimental}

The proposed model is compared with Convolutional Neural Network by using AlexNet as a pre-trained CNN [15]. AlexNet contains majorly eight layers with weights, the first five are convolutional layers and the remaining three are fully connected layer, which are fed to a 1000-way softmax layer. The softmax layer produces the distribution over the 1000 class labels. Relu is applied after each convolutional and fully connected layer [29, 30]. Table 2 illustrates the accuracy of vein palm image classification based on AlexNet.

Four classifiers are applied to predict the palm vein data. These classifiers are KNN, SVM, NB, and DT. Each classifier is applied individually on raw features, reduction features based on PCA, and with feature selection by wrapper model. In $\mathrm{KNN}$, the number of neighbors $(K)$ has been used in evaluating the performance accuracy of the mentioned models and is set by one in order to reduce the noise (increase the probability of close neighbor) of the classifier. The features extraction by 2D-DWT is with high redundancy, therefore, the application of classifiers gave relatively non-high results. In order to prove the accuracy of features selected by PSO, the algorithms run thirty times and the mean of best results are calculated for each algorithm.

Table 2. Accuracy of Palm vein image classification based on AlexNet

\begin{tabular}{|c|c|c|}
\hline Algorithm & Dataset & Accuracy \\
\hline AlexNet & Left hand & $63.50 \%$ \\
\hline AlexNet & Right hand & $62.50 \%$ \\
\hline
\end{tabular}

Table 3. Accuracy of palm image classification based on 2D DWT

\begin{tabular}{|c|c|c|c|c|c|c|}
\hline \multirow{2}{*}{ 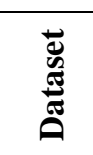 } & \multirow[b]{2}{*}{ Uీ } & \multirow{2}{*}{ 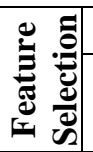 } & \multicolumn{4}{|c|}{ Accuracy } \\
\hline & & & $K N N$ & $S V M$ & $N B$ & $D T$ \\
\hline $\begin{array}{l}\text { Left } \\
\text { hand }\end{array}$ & No & No & 61.89 & 74.29 & 72.18 & 70.89 \\
\hline $\begin{array}{l}\text { Right } \\
\text { hand }\end{array}$ & No & No & 58.63 & 78.79 & 74.41 & 71.65 \\
\hline $\begin{array}{l}\text { Left } \\
\text { hand }\end{array}$ & Yes & No & 78.16 & 82.47 & 80.64 & 78.48 \\
\hline $\begin{array}{l}\text { Right } \\
\text { hand }\end{array}$ & Yes & No & 80.12 & 83.51 & 80.55 & 77.37 \\
\hline $\begin{array}{l}\text { Left } \\
\text { hand }\end{array}$ & No & Yes & 84.13 & 92.85 & 90.74 & 87.04 \\
\hline $\begin{array}{l}\text { Right } \\
\text { hand }\end{array}$ & No & Yes & 83.94 & 91.02 & 89.12 & 85.94 \\
\hline $\begin{array}{l}\text { Left } \\
\text { hand }\end{array}$ & Yes & Yes & 89.22 & 98.65 & 93.34 & 88.00 \\
\hline $\begin{array}{l}\text { Right } \\
\text { hand }\end{array}$ & Yes & Yes & 89.10 & 98.40 & 92.74 & 87.19 \\
\hline
\end{tabular}

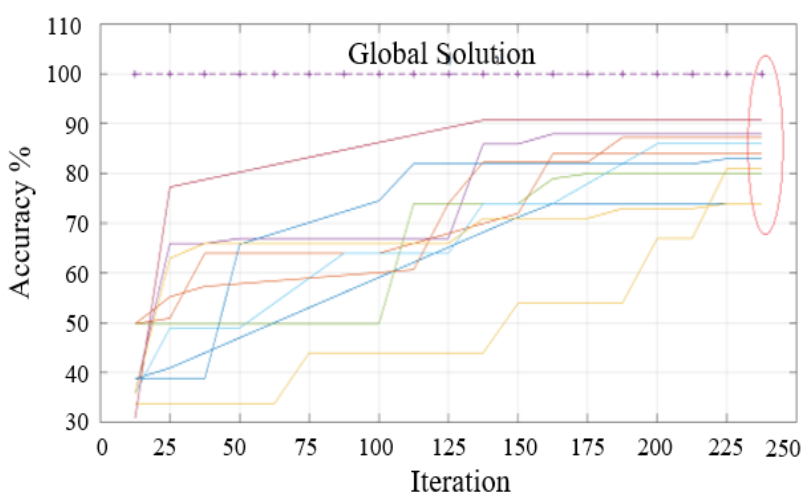

Figure. 7 PSO search without PCA (object function is SVM)

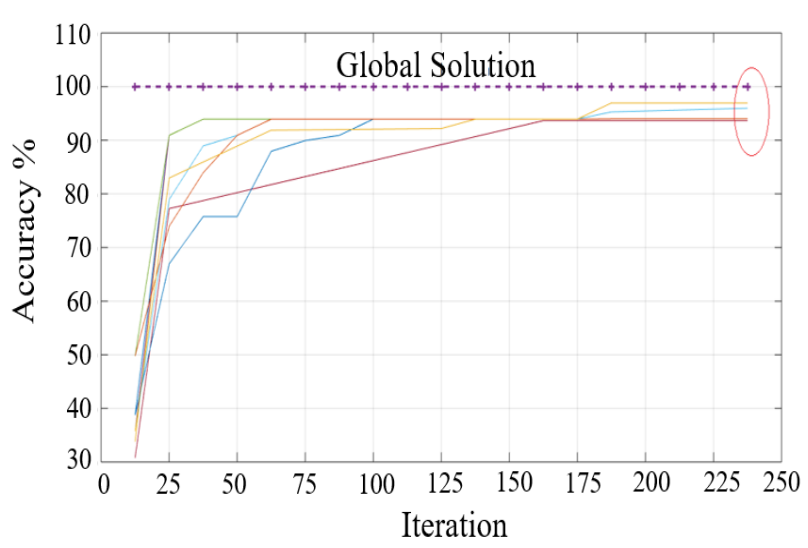

Figure. 8 PSO search with PCA (the object function is SVM)

Fig. 7 shows the PSO search of the useful and unique features from all features set, without using PCA. As shown in the figure the search problem suffering from the randomness and the selection process will not achieve higher accuracy. Whereas, fig. 8 shows the selection features from reduction features set close to 100 percentage, and achieve good result compare with other methods of a classification as shown in Table 3.

The SVM achieves the best result compared with other models when reduction features by PCA and selected feature by PSO because both SVM and PCA have the same principles in splitting data, where both algorithms build their model based on vectorizing data [24].

The KNN gets the lowest result because it relies on the convergence data. Clearly, the proposed model has significant enhancing on classifier accuracy. In other words, the proposed 2D-DWTPP model has delivered accurate results in term of classification of palm vein image datasets. Moreover, the results show that the proposed model has indicated as a potential alternative of VPI. Additionally, our model (2DDWTPP) works to improve the performance of the algorithm automated learning because it processes 


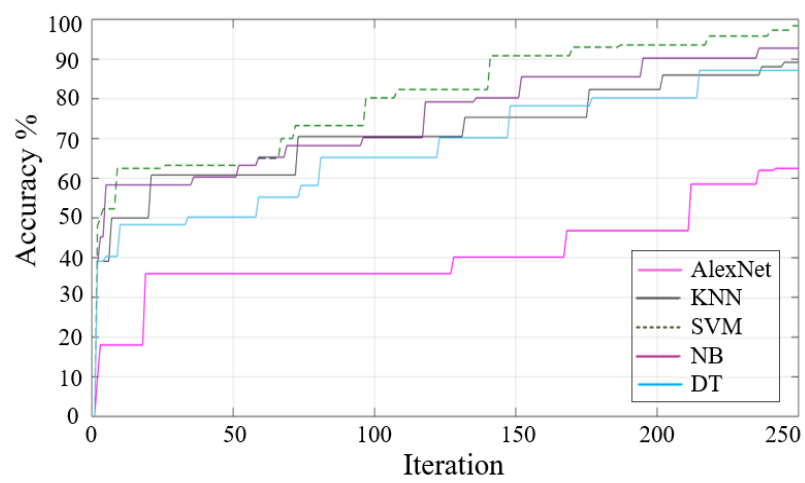

Figure. 9 Learning rate of AlexNet and 2D-DWTPP with (SVM, KNN, NB, DT)

Table 4. Summary of comparison

\begin{tabular}{|l|l|l|}
\hline \multicolumn{1}{|c|}{ Algorithm } & \multicolumn{1}{c|}{ Dataset } & Accuracy \\
\hline$[2]$ & PUT vein images & 94 \\
\hline$[14]$ & $\begin{array}{l}\text { PUT vein images } \\
\text { (implemented to } \\
\text { be compare) }\end{array}$ & 95.6 \\
\hline$[15]$ & PUT vein images & 84 \\
\hline Proposed model & PUT vein images & 98.65 \\
\hline
\end{tabular}

features by reducing the redundancy in data before feature selection process as shown in Fig. 9.

This allows machine learning algorithms to deal with features that have high precision and relevance with object data. The proposed 2D-DWTPP has remarkably enhanced the performance of supervised machine learning algorithms (SVM, KNN, NB, DT) compering with AlexNet.

The proposed system compared two studies on palm vein identification, table 4 shows the summary of comparison for different techniques on the same dataset in terms of accuracy

Generally, the results show that the performance evaluation of proposed solution outperformed the other models in Table 4, where all the testing cases reflect a significant achievement. In other words, the proposed 2D-DWTPP model has consistently required fewer number of features to reach high accurate classification results with high-dimensional datasets, demonstrating that it is a potential alternative to VPI.

\subsection{Results discussion}

The experimental results show that our proposed method is better than other methods that are worked based on geometric and appearance - based features. In spite of that, the features of geometric are difficult to be extracted and compared, whereas the geometric features such as texture energy is not enough for palm vein recognition. The Gabor filter method can successfully recognize local changes of the force signals that is reflect the different data shape of the palm vain, therefore achieves better performance of palm vein pattern recognition.

The method in [2, 14] apply texture-based to represent of palm vein, such as VeinCode (where features are extracted by the 2-Gabor filter), thus it aims to provide the optimized resolution in both the locative and frequency domains.

The method in [15] utilizes appearance - based to enhance the palm vein image, where features are extracted by the DWT, the DWT aims to decompose the high frequency and low frequency components to enhance the poor-quality images of palm vein.

The proposed model uses local and global-based approach to reduce the redundancy in palm vein features, where features are extracted by the 2D-DWT, the 2D-DWT aims to extract features and uses the PCA to reduce the redundancy in the palm vein features. According to the aforementioned, their methods did not effectively discover the local and global features which have an important role of palm vein image pattern recognition.

\section{Conclusion and future work}

In this paper, we proposed the Palm Vein verification model using 2D Haar-Discrete Wavelet transform for features extraction. In addition, PCA was used for features reduction. For the feature selection process, Particle Swarm Optimization (PSO) was used. Moreover, we have used two-level decomposition of wavelet. The classification accuracy results of various machine learning algorithms were compared as well as different algorithms for features extraction. From the experiments, the PCA and PSO significantly improved the performance of classification. The features extracted by the $2 \mathrm{D}$ Discrete Wavelet transform have high redundancy. It is not appropriate to implement feature selection algorithms directly because they may choose recurring attributes that in turn negatively affect system performance. Therefore, it is necessary to implement the feature reduction algorithm as well as selection to increase the quality of the produced features. PUT images dataset was used in this model to test the performance accuracy of the proposed model with SVM classifier achieving accuracy of $98.65 \%$. In the future, we plan to investigate and improve features extraction techniques and feature selection process based on PSO with a minimal computation time and improved recognition accuracy.

\section{Conflicts of Interest}

The authors declare no conflict of interest. 


\section{Author Contributions}

In this research papers, individual authors have their contributions and responsibility as following: conceptualization and methodology, A. Alfoudi, A. Alsaeedi, M. Abed, and A. Otebolaku; data validation/curation, A. Alsaeedi and M. Abed; result analysis and conclusion, A. Alfoudi, A. Alsaeedi, A. Otebolaku and Y. Razooqi; writing, original draft preparation, review and editing, A. Alfoudi, A. Otebolaku and Y. Razooqi.

\section{References}

[1] M. Watanabe, T. Endoh, M. Shiohara, and S. Sasaki, "Palm vein authentication technology and its applications", In: Proc. of the Biometric Consortium Conference, pp. 19-21, 2005.

[2] M. H. Abed, "Wrist and Palm Vein pattern recognition using Gabor filter", J. AL-Qadisiyah Comput. Sci. Math., Vol. 9, No. 1, pp. 49-60, 2017.

[3] F. Ahmad, L. M. Cheng, and A. Khan, "Lightweight and privacy-preserving template generation for palm-vein-based human recognition", IEEE Trans. Inf. Forensics Secur., Vol. 15, pp. 184-194, 2019.

[4] A. M. A. juboori, W. Bu, X. Wu, and Q. Zhao, "Palm vein verification using multiple features and isometric projection", Int. J. Signal Process. Image Process. Pattern Recognit., Vol. 7, No. 1, pp. 33-44, 2014.

[5] M. K. R. Kowalski, "Human vein pattern segmentation from low quality images--a comparison of methods", Image Processing and Communications Challenges 2, pp. 105-112, 2010.

[6] M. Meddad, C. Moujahdi, M. Mikram, and M. Rziza, "A Hybrid Face Identification System using a Compressed CNN in a Big Data Environment for Embedded Devices", Int. J. Comput. Digit. Syst., Vol. 9, No. 4, pp. 689-701, 2020.

[7] R. Hernández-Garcl'lia, R. J. Barrientos, C. Rojas, and M. Mora, "Individuals identification based on palm vein matching under a parallel environment", Appl. Sci., Vol. 9, No. 14, p. 2805, 2019.

[8] N. F. Hassan and H. I. Abdulrazzaq, "Pose invariant palm vein identification system using conVolutional neural network", Baghdad Sci. J., Vol. 15, No. 4, 2018.

[9] R. Meitram and P. Choudhary, "Palm vein recognition based on 2D Gabor filter and artificial neural network", J. Adv. Inf. Technol. Vol, Vol. 9, No. 3, 2018.
[10] A. F. Akbar, T. A. B. Wirayudha, and M. D. Sulistiyo, "Palm vein biometric identification system using local derivative pattern", In: Proc. of 2016 4th International Conference on Information and Communication Technology (ICoICT), 2016, pp. 1-6.

[11] H. Setiawan and E. M. Yuniarno, "Biometric Recognition Based on Palm Vein Image Using Learning Vector Quantization", In: Proc. of 2017 5th International Conference on Instrumentation, Communications, Information Technology, and Biomedical Engineering (ICICI-BME), 2017, pp. 95-99.

[12] Y. Zhou and A. Kumar, "Human identification using palm-vein images", IEEE Trans. Inf. forensics Secur., Vol. 6, No. 4, pp. 1259-1274, 2011.

[13] S. Bharathi and R. Sudhakar, "Biometric recognition using finger and palm vein images", Soft Comput., Vol. 23, No. 6, pp. 1843-1855, 2019.

[14] S. Elnasir and S. M. Shamsuddin, "Palm vein recognition based on 2D-discrete wavelet transform and linear discrimination analysis", Int. J. Adv. Soft Compu. Appl, Vol. 6, No. 3, pp. 2074-8523, 2014.

[15] S. Lefkovits, L. Lefkovits, and L. Szilágyi, "Applications of different $\mathrm{CNN}$ architectures for palm vein identification", In: Proc. of International Conference on Modeling Decisions for Artificial Intelligence, pp. 295-306, 2019.

[16] W. Alexan, M. E. Beheiry, and O. G. Eldin, "A comparative study among different mathematical sequences in $3 \mathrm{~d}$ image steganography", Int. J. Comput. Digit. Syst., Vol. 9, No. 4, pp. 545-552, 2020.

[17] Q. A. A. Hadi, "Vein palm recognition model using fusion of features", Telkomnika, Vol. 18, No. 6, pp. 2921-2927, 2020.

[18] A. Kumar, M. Hanmandlu, and H. M. Gupta, "Hybrid PSO based integration of multiple representations of thermal hand vein patterns", In: proc. of International Conference on Swarm, EVolutionary, and Memetic Computing, pp. 246-253, 2010.

[19] M. S. M. M. Patil, "Interpolation Techniques in Image Resampling”, Int. J. Eng. Technol., Vol. 7, No. 3.34, pp. 567-570, 2018.

[20] A. Sophian, G. Y. Tian, D. Taylor, and J. Rudlin, "A feature extraction technique based on principal component analysis for pulsed Eddy current NDT", NDT e Int., Vol. 36, No. 1, pp. 37-41, 2003. 
[21] A. R. Kalukin, M. V. Geet, and R. Swennen, "Principal components analysis of multienergy $\mathrm{X}$-ray computed tomography of mineral samples", IEEE Trans. Nucl. Sci., Vol. 47, No. 5, pp. 1729-1736, 2000.

[22] G. Chandrashekar and F. Sahin, "A survey on feature selection methods", Comput. Electr. Eng., Vol. 40, No. 1, pp. 16-28, 2014.

[23] R. Eberhart and J. Kennedy, "A new optimizer using particle swarm theory", In: Proc. of MHS'95. Proceedings of the Sixth International Symposium on Micro Machine and Human Science, pp. 39-43, 1995.

[24] J. Zhu, W. Lu, L. Liu, T. Gu, and B. Niu, "Classification of Src kinase inhibitors based on support vector machine", QSAR Comb. Sci., Vol. 28, No. 6-7, pp. 719-727, 2009.

[25] A. H. A. Saeedi, O. Altun, and others, "Binary mean-variance mapping optimization algorithm (BMVMO)", J. Appl. Phys. Sci., Vol. 2, No. 2, pp. 42-47, 2016.

[26] S. R. Safavian and D. Landgrebe, "A survey of decision tree classifier methodology", IEEE Trans. Syst. Man. Cybern., Vol. 21, No. 3, pp. 660-674, 1991.

[27] A. M. Taha, A. Mustapha, and S. D. Chen, "Naive Bayes-guided bat algorithm for feature selection", Sci. World J., Vol. 2013, 2013.

[28] M. K. R. Kowalski, "Human vein pattern correlation-a comparison of segmentation methods", In: Proc. of Computer Recognition Systems 4, pp. 51-59, 2011.

[29] M. H. Abed, A. H. I. A. Rammahi, and M. J. Radif, "Real-Time Color Image Classification Based On Deep Learning Network", J. Southwest Jiaotong Univ., Vol. 54, No. 5, 2019.

[30] M. A. Kadhim and M. H. Abed, "ConVolutional neural network for satellite image classification", In: Proc. of Asian Conference on Intelligent Information and Database Systems, pp. 165-178, 2019. 